\title{
Editorial: Peer-to-peer networking and applications, volume 2, issue 2
}

\author{
Xuemin (Sherman) Shen • Heather Yu
}

Published online: 14 April 2009

(C) Springer Science + Business Media, LLC 2009

This issue of the Peer-to-Peer Networking and Applications includes six original research works which address research problems related to network traffic classification, search, modeling networks with generous peers, XML data management, service differentiation and trust.

A significant portion of today's Internet traffic is P2P traffic. As a result, communication service providers are often interested in classifying the traffic. The traditional traffic identification techniques perform poorly in their job, due to dynamics of $\mathrm{P} 2 \mathrm{P}$ applications. In the first paper of this issue, "Exploiting Unlabeled Data to Improve Peer-to-Peer Traffic Classification Using Incremental Tri-Training Method", Raahemi et al. propose a incremental Tri-Training based semi-supervised learning method to classify $\mathrm{P} 2 \mathrm{P}$ traffic. In contrast to controversial deep packet inspection technique, this method simply relies on IP headers to identify P2P traffic.

Search in unstructured P2P network is challenging. Hub nodes, having a large number of connections, and generous nodes, sharing many resources, significantly influence performance of information search over unstructured P2P networks. In the second paper, "Effi-

\footnotetext{
X. Shen $(\varangle)$

Department of Electrical and Computer Engineering,

University of Waterloo, Waterloo, Ontario,

Canada N2L 3G1

e-mail: xshen@bbcr.uwaterloo.ca

H. Yu

Huawei Technologies USA,

Bridgewater, NJ, USA

e-mail: heathery@ieee.org
}

cient and Scalable Search on Scale-free P2P Networks", Liu et al. present a new search method for unstructured network called Scalable Peer-to-Peer Search or SP2PS. Unlike existing searches, SP2PS uses more than one parameter to forward search queries.

Though a good portion of participants of a typical $\mathrm{P} 2 \mathrm{P}$ network do not contribute, $\mathrm{P} 2 \mathrm{P}$ networks are thriving in the Internet. The reason for this vivid survival is a few altruistic peers. In An Analysis of Peer-to-Peer Networks with Altruistic Peers, Vassilakis and Vassalos develop a new model of the interaction of rational peers in a P2P network with altruistic peers. The goal is to have a better understanding of the reasons for survival of $\mathrm{P} 2 \mathrm{P}$ networks even without any incentive mechanism.

$\mathrm{XML}$ is a well accepted self descriptive language to describe information. XML is widely used to exchange data. In the fourth paper, "XCube: Processing XPath Queries in a Hypercube Overlay Network", Li et al. present the design of XCube, a tag-based system for managing XML data in a hypercube overlay network. A query mechanism and a dynamic partitioning mechanism for load balancing are also introduced.

One way to improve quality of service in a $\mathrm{P} 2 \mathrm{P}$ system is service differentiation. It is important to identify both free riders and malicious peers, and to reduce the level of service provided to them, and at the same, to ensure better services for good peers. In $A$ Contribution-Based Service Differentiation Scheme for Peer-to-Peer Systems, Mekouar et al. develop a new service differentiation mechanism. In this research, both availability and involvement are used to differentiate peers.

The concept of software as service has received a tremendous interest from researchers and indus- 
tries. Several solutions propose to provide dynamic cross organization service to increase productivity and usability. However, when different organizations are considered, support for common security platform lacks. In Context-Aware Trust Negotiation in Peer-toPeer Service Collaborations, Li et al. proposes a new policy to establish trust among such organizations in a peer-to-peer way.

In closing, we would like to acknowledge the contributions of the authors who submitted their works and the reviewers who provided their expert opinions and constructive and helpful comments. We would also like to extend our sincere thanks to Melissa Fearon, the Senior Editor, Valerie Schofield, and Cristina Chua of Springer, for their support and help in bringing forward the second issue of this volume. We hope that you will enjoy the current issue and find the journal useful.

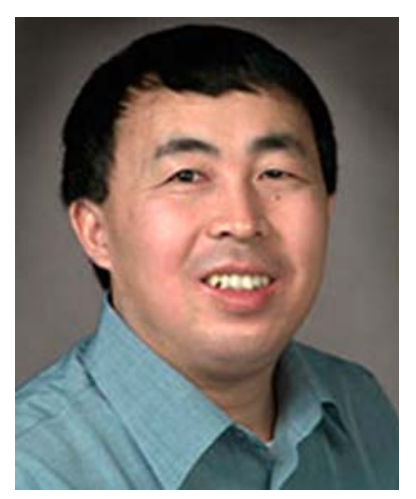

Xuemin (Sherman) Shen (IEEE M'97-SM'02-F'09) received the B.Sc. (1982) degree from Dalian Maritime University (China) and the M.Sc. (1987) and Ph.D. degrees (1990) from Rutgers University, New Jersey (USA), all in electrical engineering. He is a Professor and University Research Chair, Department of Electrical and Computer Engineering, University of Waterloo, Canada. Dr. Shen's research focuses on mobility and resource management in interconnected wireless/wired networks, UWB wireless communications networks, wireless network security, wireless body area networks and vehicular ad hoc and sensor networks. He is a co-author of three books, and has published more than 400 papers and book chapters in wireless communications and networks, control and filtering. Dr. Shen served as the Tutorial Chair for IEEE ICC'08, the Technical Program Committee Chair for IEEE Globecom'07, the General Co-Chair for Chinacom'07 and QShine'06, the Founding Chair for IEEE Communications Society Technical Committee on P2P Communications and Networking. He also serves as a Founding Area Editor for IEEE Transactions on Wireless Communications; Editor-in-Chief for Peer-to-Peer Networking and Application; Associate Editor for IEEE Transactions on Vehicular Technology; KICS/IEEE Journal of Communications and Networks, Computer Networks; ACM/Wireless Networks; and Wireless Communications and Mobile Computing (Wiley), etc. He has also served as Guest Editor for IEEE JSAC, IEEE Wireless Communications, IEEE Communications Magazine, and ACM Mobile Networks and Applications, etc. Dr. Shen received the Excellent Graduate Supervision Award in 2006, and the Outstanding Performance Award in 2004 and 2008 from the University of Waterloo, the Premier's Research Excellence Award (PREA) in 2003 from the Province of Ontario, Canada, and the Distinguished Performance Award in 2002 and 2007 from the Faculty of Engineering, University of Waterloo. Dr. Shen is a registered Professional Engineer of Ontario, Canada, and a Distinguished Lecturer of IEEE Communications Society.

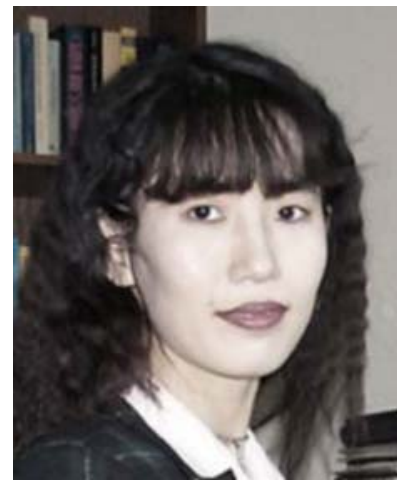

Heather Yu received my Ph.D. in Electrical Engineering from Princeton University in 1998. From September 1998 to March 2007, she was with Panasonic Princeton Laboratory where her major focus was multimedia communication and multimedia security R\&D. Currently she is the Senior Manager of Media Technologies at Huawei Technologies USA. One of her responsibilities is to lead the establishment of the multimedia content networking research team in New Jersey, USA. Her current research interests include human centric multimedia communications, IPTV, Peer-to-peer networking, mobile multimedia, etc. She is serving as the Associate Editor-in-Chief of the Journal of Peer-to-Peer Networking and Applications, Associate Editor of IEEE Multimedia Magazine and IEEE Communications Surveys and Tutorials, and Chair of the Human Centric Technical Subcommittee of IEEE ComSoc. She served as Technical Program Chair for several IEEE multimedia and communications conferences, the Chair of IEEE Multimedia Communications Technical Committee, and a voting member at ComSoc GITC, ComSoc SPC(strategic planning committee), etc. She has published 2 books, more than 60 technical papers and holds 23 US patents. 